Pacific Journal of Mathematics

ON $g$-METRIZABILITY 


\title{
ON g-METRIZABILITY
}

\section{FOGED}

\begin{abstract}
We show that a regular topological space is $g$-metrizable if and only if it is weakly first countable and admits a $\sigma$ locally finite $k$-network and that a $g$-metrizable space need not be $g$-developable.
\end{abstract}

o. Introduction. G-metrizable spaces were defined in [8], where it was also shown that a space admits a countable weak base if and only if it is weakly first countable and has a countable $k$ network. In this paper we provide the corresponding result for $g$ metrizable spaces and give an example of a $g$-metrizable space which is not $g$-developable. The former result is in response to a question in [8], the latter answers a question in [6]. All spaces are at least regular.

\section{Definition.}

1.1. Let $X$ be a space. If $\Gamma$ is a family of subsets of $X$ and $\zeta: \Gamma \rightarrow \mathscr{P}(X)$ is a function, then the pair $\langle\Gamma, \zeta\rangle$ is a weak base for $X$ if, in addition, the following hold:

(a) For every member $G$ of $\Gamma, \zeta(G)$ is a subset of $G$.

(b) If $G_{1}$ and $G_{2}$ are members of $\Gamma$ and $x$ is an element of $\zeta\left(G_{1}\right) \cap \zeta\left(G_{2}\right)$, then there is a member $G_{3}$ of $\Gamma$ so that $x$ is in $\zeta\left(G_{3}\right)$ and $G_{3}$ is a subset of $G_{1} \cap G_{2}$.

(c) A subset $U$ of $X$ is open if and only if for every element $x$ of $U$ there is a member $G$ of $\Gamma$ so that $x$ is in $\zeta(G)$ and $U$ contains. $G$.

This definition of weak base differs from that of [1], namely, a collection $\mathscr{B}=U\left\{T_{x}: x \in X\right\}$ is a weak base for $X$ if a set $U$ is open in $X$ precisely when for each point $x \in U$ there exists $B \in T_{x}$ such that $B \subset U$. It is easy to see that our definition is equivalent to this, for if $B$ is as above, we let $\Gamma=\mathscr{B}$ and for $G \in \Gamma$, let $\delta(G)=$ $\left\{x: G \in T_{x}\right\}$ and if $\langle\Gamma, \delta\rangle$ is a weak base by 1.1 , then we let $T_{x}=$ $\{G: x \in \delta(G)\}$ and $\mathscr{B}=\cup\left\{T_{x}: x \in X\right\}$.

1.2. A space $X$ is $g$-metrizable if it has a weak base $\langle\Gamma, \zeta\rangle$ where $\Gamma$ is a $\sigma$-locally finite family. $X$ is weakly first countable if $X$ has a weak base $\langle\Gamma, \zeta\rangle$ so that the family $\{\zeta(G): G \in \Gamma\}$ is point countable or, equivalently, there is a function $B: \omega \times X \rightarrow \mathscr{P}(X)$ (called a wfc system for $X$ ) so that

(a) for all $n<\omega$ and $x \in X, B(n+1, x) \subset B(n, x)$;

(b) for all $x$ in $X, x \in \cap\{B(n, x): n<\omega\}$ 
(c) a subset $U$ of $X$ is open if and only if for every $x$ in $U$ there is an $n<\omega$ so that $U$ contains $B(n, x)$.

If $x$ is an element of a space $X$, then a subset $S$ of $X$ is said to be weak neighborhood of $x$ if every sequence converging to $x$ is eventually in $S$. One may show that if $X$ is weakly first countable with weak base $\langle\Gamma, \zeta\rangle$ so that $\{\zeta(G): G \in \Gamma\}$ is point countable, then $S$ is a weak neighborhood of $x$ if and only if $S$ contains a member $G$ of $\Gamma$ so that $x \in \zeta(G)$. Thus weakly first countable spaces are sequential [4].

1.3. If $X$ is a space, a collection $\Gamma$ of subsets of $X$ is said to be a $k$-network [7] for $X$ if for any compact subset $K$ of $X$ and any neighborhood $U$ of $K$, there is a finite subcollection $\Gamma^{\prime}$ of $\Gamma$ so that $K \subset \cup \Gamma^{\prime} \subset U$.

\section{2. $g$-metrizability and $k$-networks.}

LEMMA 2.1. If $X$ is a space in which points are $G_{\dot{o}}$ and if $\langle\Gamma, \zeta\rangle$ is a weak base for $X$, then $\Gamma$ is a k-network for $X$.

Proof. Let $K$ be a compact subset of $X$ and $U$ an open neighborhood of $K$. As $K$ is closed, $\left\langle\Gamma^{\prime}, \zeta^{\prime}\right\rangle$ given by $\Gamma^{\prime}=\{G \cap K: G \in \Gamma\}$ and $\zeta^{\prime}(G \cap K)=\zeta(G) \cap K$ for all $G$ in $\Gamma$, is a weak base for $K$. Thus since $K$ is Fréchet, for every $G$ in $\Gamma \zeta^{\prime}(G \cap K) \subset \operatorname{int}_{K}(G \cap K)$. Consequently if $\Gamma^{*}$ is a subcollection of $\Gamma$ so that $K \subset \cup\left\{\zeta(G): G \in \Gamma^{*}\right\}$ and $U \Gamma^{*} \subset U$, then a finite subfamily of $\Gamma^{*}$ convers $K$.

TheORem 2.2 [3]. A regular space with a $\sigma$-locally finite $k$ network has a $\sigma$-dicrete k-network.

Lemma 2.3. Suppose $X$ hase $\langle\Gamma, \zeta\rangle$ so that $\Gamma=\cup\left\{\Gamma_{n}: n<\omega\right\}$ where every $\Gamma_{n}$ ts a closure-preserving family of closed sets. If $\left\{F_{\alpha}: \alpha \in I\right\}$ is a discrete collection of subsets of $X$, then there is a pairwise disjoint collection $\left\{N_{\alpha}: \alpha \in I\right\}$ so that for every $\alpha \in I$ and $x \in F_{\alpha}$, there is a $G$ in $\Gamma$ so that $x \in \zeta(G)$ and $G \subset N_{\alpha}$.

Proof. For each $n<\omega$ and each $\alpha \in I$, let

$$
G(n, \alpha)=\bigcup\left\{G \in \Gamma_{n}: G \cap\left(\cup\left\{F_{\beta}: \beta \neq \alpha\right\}\right)=\varnothing\right\}
$$

For each $\alpha \in I$, let

$$
N_{\alpha}=\bigcup_{n<\omega}[G(n, \alpha) \backslash \cup\{G(m, \beta): m \leqq n, \beta \neq \alpha\}] .
$$

Of course $\left\{N_{\alpha}: \alpha \in I\right\}$ is pairwise disjoint; we now verify that $\left\{N_{\alpha}: \alpha \in I\right\}$ is the desired collection. Let $\alpha \in I$ and let $x \in F_{\alpha}$. Find an 
$n<\omega$ and a $G_{1}$ in $\Gamma_{n}$ so that $x \in \zeta\left(G_{1}\right)$ and so that $G_{1}$ misses the closed set $\cup\left\{F_{\beta}: \beta \neq \alpha\right\}$. Pick $G_{2} \in \Gamma$ so that $x \in \zeta\left(G_{2}\right)$ and so that $G_{2}$ misses the closed set $\cup\{G(m, \beta): m \leqq n, \beta \neq \alpha\}$. Now there is a $G_{3} \in \Gamma$ with $x \in \zeta\left(G_{3}\right)$ so that $G_{3}$ is a subset of $G_{1} \cap G_{2}$, hence $G_{3} \subset N_{\alpha}$, as desired.

We are now in a position to prove the main result of this section.

TheOREM 2.4. A regular space is g-metrizable if and only if it is weakly first countable and admits a $\sigma$-locally finite k-network.

Proof. The necessity follows from Lemma 2.1. For the sufficiency: by Theorem 2.2, for each $n<\omega$ let $\Lambda_{n}$ be a discrete collection of closed subsets of $X$ so that $A=\cup\left\{A_{n}: n<\omega\right\}$ is closed under finite intersections and is a $k$-network for $X$. Let

$$
\Gamma=\left\{\cup \Lambda^{*}: \Lambda^{*} \text { is a finite subset of } \Lambda \text { so that } \cap \Lambda^{*} \neq \varnothing\right\} \text {. }
$$

For $\Lambda^{*}$ a finite subset of $\Lambda$ with $\cap \Lambda^{*} \neq \varnothing$, let

$$
\zeta\left(\cup \Lambda^{*}\right) \simeq\left\{x \in \cap \Lambda^{*}: \cup \Lambda^{*} \text { is a weak neighborhood of } x\right\} \text {. }
$$

Note that $\{(G): G \in \Gamma\}$ is point-countable. We now show that $\langle\Gamma, \zeta\rangle$ is a weak base for $X$. One easily verifies that (a) and (b) of 1.1 are satisfied. For (c), observe that if $U$ is a subset of $X$ so that for every $x \in U$ there is a $G \in \Gamma$ so that $x \in \zeta(G)$ and $U$ contains $G$, then $U$ is sequentially open, hence open. Conversely, suppose $U$ is open and there is an element $x$ of $U$ so that $U$ contains no member $G$ of $\Gamma$ such that $x \in \zeta(G)$, i.e. the union of no finite subset of $\left\{L_{j}: j<\omega\right\}=\{L \in \Lambda: x \in L, L \subset U\}$ is a weak neighborhood of $x$. Let $B$ a wfe system for $X$ so that $B(1, x) \subset U$. Inductively pick a sequence $\left\{x_{n}: n<\omega\right\}$ so that $x_{n} \in B(n, x) \backslash \cup\left\{L_{j}: j \leqq n\right\}$. The sequence $\left\{x_{n}: n<\omega\right\}$ converges to $x$, hence $\{x\} \cup\left\{x_{n}: n<\omega\right\}$ is compact. Let $\Lambda^{\prime}$ be a finite subset of $\Lambda$ so that $\{x\} \cup\left\{x_{n}: n<\omega\right\} \subset \cup \Lambda^{\prime} \subset U$ and let $\Lambda^{*}=\left\{L \in \Lambda^{\prime}: x \in L\right\}$. The closed set $\cup\left(\Lambda^{\prime} \backslash \Lambda^{*}\right)$ omits $x$, so there is an $m<\omega$ so that $\{x\} \cup\left\{x_{n}: n \geqq m\right\} \subset \cup \Lambda^{*}$. Also $\Lambda^{*} \subset\{L \subset \Lambda: x \in L, L \subset U\}$, so there is an $r \geqq m$ so that $\Lambda^{*} \subset\left\{L_{j}: j \leqq r\right\}$, which implies that $x_{r} \in \cup \Lambda^{*} \subset \cup\left\{L_{j}: j \leqq r\right\}$. This contradicts the fact that $x_{r}$ was picked in the complement of $U\left\{L_{j}: j \leqq r\right\}$. Thus if $U$ is open, then for all $x \in U, U$ contains a $G \in \Gamma$ so that $x \in \zeta(G)$; so $\langle\Gamma, \zeta\rangle$ is a weak base for $X$.

Note that if $n<\omega$, $\Gamma_{n}=\left\{\cup \Lambda^{*}: \Lambda^{*}\right.$ is a finite subset of $\cup\left\{\Lambda_{j}: j \leqq n\right\}$ so that $\left.\cap \Lambda^{*} \neq \varnothing\right\}$ is a closure-preserving collection, hence $\Gamma=\cup\left\{\Gamma_{n}: n<\omega\right\}$ is $\sigma$-conservative. 
For every finite subset $S$ of $\omega$, let

$$
\Lambda_{S}=\left\{\Lambda^{*}: \text { for } n<\omega \Lambda^{*} \cap \Lambda_{n} \neq \varnothing \text { iff } n \in S ; \cap \Lambda^{*} \neq \varnothing\right\}
$$

and write $\Lambda_{S}=\left\{\Lambda_{\alpha}^{*}: \alpha \in I(S)\right\}$. Further, as $\left\{\cap \Lambda_{\alpha}^{*}: \alpha \in I(S)\right\}$ is a discrete collection, use Lemma 2.3 to find a pairwise disjoint collection $\left\{N_{\alpha}\right.$ : $\alpha \in I(S)\}$ so that for every $\alpha$ in $I(S) N_{\alpha}$ is a weak neighborhood of $\cap \Lambda_{\alpha}^{*}$.

Now if $n<\omega, S$ is a finite subset of $\omega$, and if $\alpha \in I(S)$, let

$$
G(n, \alpha)=\cup\left\{G \in \Gamma_{n}: G \subset\left(\cup \Lambda_{\alpha}^{*}\right) \cap N_{\alpha}\right\} .
$$

and let

$$
\zeta^{\prime}(G(n, \alpha))=\cup\left\{\zeta(G) \cap \zeta\left(\cup \Lambda_{\alpha}^{*}\right): G \in \Gamma_{n}, G \subset\left(\cup \Lambda_{\alpha}^{*}\right) \cap N_{\alpha}\right\} .
$$

If $n<\omega$ and if $S$ is a finite subset of $\omega$, let

$$
\Gamma(n, S)=\{G(n, \alpha): \alpha \in I(S)\} .
$$

The collections $\Gamma(n, S)$ are conservative and, since $G(n, \alpha) \subset N_{\alpha}$ for every $\alpha \in I(S)$, pairwise disjoint, hence discrete. Let $\Gamma^{\prime}$ be the family of all intersections of finite subcollections of $\cup\{\Gamma(n, S): n<\omega$, $S$ a finite subset of $\omega\}$ and extend $\zeta^{\prime}$ to $\Gamma^{\prime}$ by $\zeta^{\prime}\left(\bigcap_{i=1}^{k} G\left(n_{i}, \alpha_{i}\right)\right)=$ $\bigcap_{i=1}^{k} \zeta^{\prime}\left(G\left(n_{i}, \alpha_{i}\right)\right)$. Observe that $\Gamma^{\prime}$ is $\sigma$-discrete; we will show that $\left\langle\Gamma^{\prime}, \zeta^{\prime}\right\rangle$ is a weak base for $X$, completing the proof.

Conditions (a) and (b) of 1.1 are easily verified. Recalling that $\{\zeta(G): G \in \Gamma\}$ is point countable, the remarks in 1.2 give that for all $G \in \Gamma G$ is a weak neighborhood of $\zeta(G)$ so that if $n<\omega, S$ is a finite subset of $\omega$ and if $\alpha \in I(S)$, then $G(n, \alpha)$ is a weak neighborhood of $\zeta^{\prime}(G(n, \alpha))$. Consequently if $G^{\prime} \in \Gamma^{\prime}$, then $G^{\prime}$ is a weak neighborhood of $\zeta\left(G^{\prime}\right)$. Hence if $U$ is a subset of $X$ such that for every member $x$ of $U$ there is a member $G^{\prime}$ of $\Gamma^{\prime}$ with $x \in \zeta\left(G^{\prime}\right)$ and $G^{\prime} \subset U$, then $U$ is a weak neighborhood of each of its elements, thus sequentially open, and so $U$ is open. To complete the proof of (c), let $U$ be an open subset of $X$, and let $x \in U$. Since $\langle\Gamma, \zeta\rangle$ is a weak base for $X$, there is a finite subset $\Lambda^{*}$ of $A$ so that $x \in \zeta\left(\cup \Lambda^{*}\right) \subset$ $\cap \Lambda^{*} \subset \cup \Lambda^{*} \subset U$. Find a finite subset $S$ of $\omega$ and an $\alpha \in I(S)$ so that $\Lambda^{*}=\Lambda_{\alpha}^{*}$. Since $\cup \Lambda_{\alpha}^{*}$ is a member of $\Gamma, \cup \Lambda_{\alpha}^{*}$ is a weak neighborhood of $\zeta\left(\cup \Lambda_{\alpha}^{*}\right)$, hence of $x ; N_{\alpha}$ is a weak neighborhood of $\cap \Lambda_{\alpha}^{*}$, hence of $x$; thus $\left(\cup \Lambda_{\alpha}^{*}\right) \cap N_{\alpha}$ is a weak neighborhood of $x$. Again since $\{\zeta(G): G \in \Gamma\}$ is point-countable, we have that there is an $n<\omega$ and a $G \in \Gamma_{n}$ so that $x \in \zeta(G)$ and $G \subset\left(\cup \Lambda_{\alpha}^{*}\right) \cap N_{\alpha}$. Thus $x \in \zeta^{\prime}(G(n, \alpha))$ and $G(n, \alpha) \subset \cup \Lambda_{\alpha}^{*} \subset U$. Thus (c) is established.

3. $g$-developable spaces. Generalizing a characterization of developability given in [5], Lee [6] defined $g$-developable spaces to 
be those weakly first countable spaces $X$ which have a wfc system satisfying the following: if $x \in X$ and if $\left\{x_{n}: n<\omega\right\}$ and $\left\{y_{n}: n<\omega\right\}$ are sequences in $X$ so that for every $n<\omega x$ and $x_{n}$ are elements of $B\left(n, y_{n}\right)$, then the sequence $\left\{x_{n}: n<\omega\right\}$ converges to $x$.

Proposition 3.1. A $\sigma$-discrete weakly first countable space $X$ is g-developable.

Proof. Write $X=\cup\left\{D_{n}: n<\omega\right\}$, where $D_{n}$ is a closed discrete set for every $n<\omega . \quad X$ is symmetrizable [1], so let $d$ be a compatible symmetric function. We define $B: w \times X \rightarrow \mathscr{P}(X)$ as follows: if $m$ and $n$ are finite ordinals and if $x \in D_{m}$, let

$$
B(n, x)=\{y \in X: d(x, y)<1 / n\} \backslash \cup\left\{D_{k}: k<m\right\} .
$$

One easily checks that $B$ is a wfe system for the topology of $X$. To see that $B$ satisfies the defining condition for $g$-developability let $x \in X$ and let $\left\{x_{n}: n<\omega\right\}$ and $\left\{y_{n}: n<\omega\right\}$ be sequences in $X$ so that for every $n<\omega x$ and $x_{n}$ are in $B\left(n, y_{n}\right)$. If $m<\omega$ so that $x \in D_{m}$, then there is a $j<\omega$ so that $\{y \in X: d(x, y)<1 / j\} \cap\left(\cup\left\{D_{k}: k \leqq m\right\}\right)=$ $\{x\}$. The fact that $x \notin \cup\{B(j, y): y \neq x\}$ implies that if $n \geqq j$, then $y_{n}=x$. Thus for all $n \geqq j$ we have $x_{n} \in B(n, x)$, hence $\left\{x_{n}: n<\omega\right\}$ converges to $x$, as desired.

The definition of $g$-developable inspires the question to which the following is a negative answer.

THEOREM 3.2. There is a g-metrizable space which is not gdevelopable.

Proof. Let $\boldsymbol{R}$ denote the set of real numbers $\boldsymbol{Q}$ the set of rationals. Choose a countable quasibase $\Lambda$ for the Euclidean topology of $\boldsymbol{R}$ consisting of closed sets. Let $X=\left\{\langle x, y\rangle \in R^{2}\right.$ : either $y=0$, or $x \in \boldsymbol{Q} \& 1 / y \in \omega\}$, and view $\boldsymbol{R}$ as $\{\langle x, y\rangle \in X: y=0\}$. For every $q \in \boldsymbol{Q}$ and $m<\omega$, define $A(m, q)=\{r \in \boldsymbol{R}:|r-q| \leqq 1 / m\} \cup\{\langle q, 1 / n\rangle$ : $n>m\}$. Let

$$
\Gamma=\{A(m, q): m<\omega, q \in \boldsymbol{Q}\} \cup \Lambda \cup\{\{\langle q, 1 / n\rangle\}: q \in \boldsymbol{Q}, n<\omega\},
$$

and define

$$
\begin{aligned}
& \zeta(A(m, q))=\{q\}, \quad \text { if } m<\omega \text { and } q \in \boldsymbol{Q} \text {; } \\
& \zeta(L)=\{r \in \boldsymbol{R} \backslash \boldsymbol{Q}: r \text { is in the Euclidean interior of } L\}, \text { if } L \in \Lambda \text {; } \\
& \zeta(\{\langle q, 1 / n\rangle\})=\{\langle q, 1 / n\rangle\}, \quad \text { if } q \in \boldsymbol{Q} \text { and } n<\omega .
\end{aligned}
$$

Give $X$ the topology for which $\langle\Gamma, \zeta\rangle$ is a weak base. Certainly $\Gamma$ is countable, so, as $X$ is easily seen to be regular, $X$ is $g$-metriza- 
ble. To show that $X$ is not $g$-developable, assume that $B$ is a wfcsystem for $X$ satisfying the defining condition for $g$-developability.

Define a function $\phi: \boldsymbol{R} \backslash \boldsymbol{Q} \rightarrow \omega$ so that if $r \in \boldsymbol{R} \backslash \boldsymbol{Q}$, then $r \notin$ $\cup\{B(\phi(r), q): q \in \boldsymbol{Q}\}$. This is possible, for if there is an $r \in \boldsymbol{R} \backslash \boldsymbol{Q}$ so that for every $n<\omega$ there is a $q_{n} \in \boldsymbol{Q}$ so that $r \in B\left(n, q_{n}\right)$, then find, for each $n<\omega$, an $x_{n} \in X \backslash \boldsymbol{R} \cap B\left(n, q_{n}\right)$. This would imply that for every $n<\omega, r$ and $x_{n}$ are in $B\left(n, q_{n}\right)$, but $\left\{x_{n}: n<\omega\right\}$ does not converge to $r$, a contradiction.

Since $\boldsymbol{R} \backslash \boldsymbol{Q}=\cup\{\{\boldsymbol{r} \in \boldsymbol{R} \backslash \boldsymbol{Q}: \phi(r) \leqq n\}: n<\omega\}$, there is an $m<\omega$ so that the Euclidean closure $\operatorname{cl}_{\boldsymbol{R}}\{r \in \boldsymbol{R} \backslash \boldsymbol{Q}: \phi(r) \leqq m\}$ contains a Euclidean open set $U$. Choose a $p \in \boldsymbol{Q} \cap U$. As $B(m, p) \cap \boldsymbol{R}$ is a Euclidean neighborhood of $p$ in $R$, there is an $r \in \boldsymbol{R} \backslash \boldsymbol{Q} \cap B(m, p)$ so that $\dot{\phi}(r) \leqq m$, that is $r \notin \cup\{B(m, q): q \in \boldsymbol{Q}\}$; this contradiction completes the proof.

\section{REFERENCES}

1. A. Arhangel'skii, Mappings and spaces, Russ. Math. Surveys, 21 (1966), no. 4, 115-162.

2. A. Arhangel'skii and S. Franklin, Ordinal invariants for topological spaces, Michigan Math. J., 15 (1968), 313-320.

3. L. Foged, Separation in k-and-\$ spaces, to appear.

4. S. Franklin, Spaces which sequence suffice, Fund. Math., 57 (1965), 107-115.

5. R. Heath, Arcwise connectedness in semi-metric spaces, Pacific J. Math., 12 (1962), 1301-1319.

6. K. Lee, On certain g-first countable spaces, Pacific J. Math., 65 (1976), 113-118.

7. E. Michael, $\boldsymbol{\aleph}_{0}$-space, J. Math. Mech., 15 (1966), 983-1002.

8. F. Siwiec, On defining a space by a weak base, Pacific J. Math., 52 (1974), 233-245.

Received February 8, 1980 and in revised form October 8, 1980.

UnIVERSITY OF TEXAS

Eu Paso, TX 79968 


\title{
PACIFIC JOURNAL OF MATHEMATICS
}

\section{EDITORS}

\author{
DONALD BABBITT (Managing Editor) \\ University of California \\ Los Angeles, CA 90024 \\ Hugo RossI \\ University of Utah \\ Salt Lake City, UT 84112 \\ C. C. MOORE and ANDREW OGG \\ University of California \\ Berkeley, CA 94720
}

J. DugundjI

Department of Mathematics

University of Southern California

Los Angeles, CA 90007

R. FinN and J. Milgram

Stanford University

Stanford, CA 94305

\section{ASSOCIATE EDITORS}
R. ARENS
E. F. Beckenbach
B. H. NeumanN
F. WOLF
K. YoSHIDA

\section{SUPPORTING INSTITUTIONS}

\author{
UNIVERSITY OF ARIZONA \\ UNIVERSITY OF BRITISH COLUMBIA \\ CALIFORNIA INSTITUTE OF TECHNOLOGY \\ UNIVERSITY OF CALIFORNIA \\ MONTANA STATE UNIVERSITY \\ UNIVERSITY OF NEVADA, RENO \\ NEW MEXICO STATE UNIVERSITY \\ OREGON STATE UNIVERSITY
}

\author{
UNIVERSITY OF OREGON \\ UNIVERSITY OF SOUTHERN CALIFORNIA \\ STANFORD UNIVERSITY \\ UNIVERSITY OF HAWAII \\ UNIVERSITY OF TOKYO \\ UNIVERSITY OF UTAH \\ WASHINGTON STATE UNIVERSITY \\ UNIVERSITY OF WASHINGTON
}

The Supporting Institutions listed above contribute to the cost of publication of this Journal, but they are not owners or publishers and have no responsibility for its content or policies.

Mathematical papers intended for publication in the Pacific Journal of Mathematics should be in typed form or offset-reproduced, (not dittoed), double spaced with large margins. Please do not use built up fractions in the text of the manuscript. However, you may use them in the displayed equations. Underline Greek letters in red, German in green, and script in blue. The first paragraph or two must be capable of being used separately as a synopsis of the entire paper. Please propose a heading for the odd numbered pages of less than 35 characters. Manuscripts, in triplicate, may be sent to any one of the editors. Please classify according to the scheme of Math. Reviews, Index to Vol. 39. Supply name and address of author to whom proofs should be sent. All other communications should be addressed to the managing editor, or Elaine Barth, University of California, Los Angeles, California, 90024.

50 reprints to each author are provided free for each article, only if page charges have been substantially paid. Additional copies may be obtained at cost in multiples of 50 .

The Pacific Journal of Mathematics is issued monthly as of January 1966. Regular subscription rate: $\$ 102.00$ a year (6 Vols., 12 issues). Special rate: $\$ 51.00$ a year to individual members of supporting institutions.

Subscriptions, orders for numbers issued in the last three calendar years, and changes of address shoud be sent to Pacific Journal of Mathematics, P.O. Box 969, Carmel Valley, CA 93924, U.S.A. Old back numbers obtainable from Kraus Periodicals Co., Route 100, Millwood, NY 10546.

PUBLISHED BY PACIFIC JOURNAL OF MATHEMATICS, A NON-PROFIT CORPORATION

Printed at Kokusai Bunken Insatsusha (International Academic Printing Co., Ltd.). 8-8, 3-chome, Takadanobaba, Shinjuku-ku, Tokyo 160, Japan. 


\section{Pacific Journal of Mathematics}

\section{Vol. 98, No. 2 \\ April, 1982}

Thomas E. Armstrong, Barycentric simplicial subdivision of infinite-dimensional simplexes and octahedra

Hom Nath Bhattarai and James William Fernandez, Joins of double coset

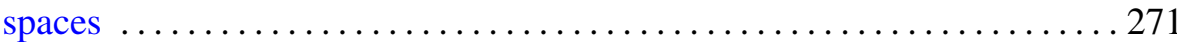

Alexandru Buium, Ritt schemes and torsion theory $\ldots \ldots \ldots \ldots \ldots \ldots \ldots 281$

Jacob Burbea, Operator-valued Pick's conditions and holomorphicity ..... 295

Su-Shing Chen, Duality condition and property $(\mathrm{S}) \ldots \ldots \ldots \ldots \ldots \ldots . \ldots . \ldots 313$

Ky Fan, Evenly distributed subsets of $S^{n}$ and a combinatorial application . . 323

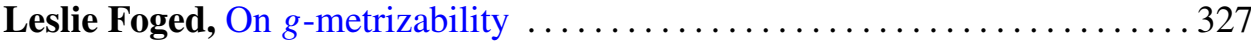

John Groves Heywood, An error estimate uniform in time for spectral

Galerkin approximations of the Navier-Stokes problem ............ 333

Aggie Ho, The Krê̆ n-Milman property and complemented bushes in

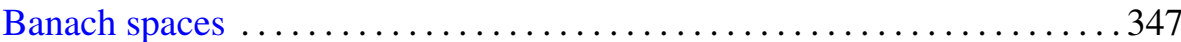

David R. Jackett, Rings on certain mixed abelian groups ............. 365

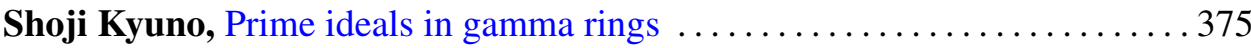

George Lucius O'Brien, Zero-inducing functions on finite abelian

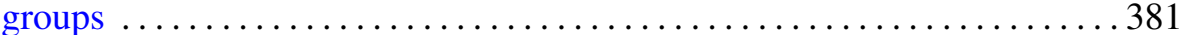

P. Robba, Sur les équations différentielles linéaires $p$-adiques. II ......... 393

Wolfgang Ruess, [Weakly] compact operators and DF spaces ...........419

Claude Schochet, Topological methods for $C^{*}$-algebras. II. Geometry resolutions and the Künneth formula

Harry F. Smith, Jr., Equivalent nilpotencies in certain generalized right

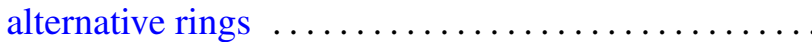

Max Warshauer, Diagonalization up to Witt 469

Hugh C. Williams, A class of primality tests for trinomials which includes the Lucas-Lehmer test 